\title{
Recent Highlights from the PHENIX Collaboration
}

\author{
Takahito Todoroki (for the PHENIX Collaboration) $)^{1, *}$ \\ ${ }^{1}$ Tomonaga Center for the History of the Universe, University of Tsukuba, Tsukuba, Ibaraki 305, Japan
}

\begin{abstract}
These proceedings present a set of PHENIX highlight results using recent high-luminosity data sets for $p+p, p\left(d,{ }^{3} \mathrm{He}\right)+\mathrm{A}$, and $\mathrm{A}+\mathrm{A}(\mathrm{B})$ collisions at RHIC top energies with a focus on: (1) light-flavor particle production; (2) hard-probes such as jet-like correlations, direct photon, and heavy-quark productions; and (3) azimuthal anisotropy in small collision systems.
\end{abstract}

\section{Introduction}

During 16 years of operations, PHENIX collected a rich amount of data for 9 collision species at several collision energies. Now the focus of the PHENIX heavy-ion program has been complete analyses of recent high-luminosity data sets for $p+p, p\left(d,{ }^{3} \mathrm{He}\right)+\mathrm{Au}(\mathrm{Al})$, as well as $\mathrm{Au}(\mathrm{Cu})+\mathrm{Au}$ and $\mathrm{U}+\mathrm{U}$ collisions at RHIC top energies. In these proceedings, we discuss the following recent highlight results from such an effort: (i) $\pi^{0}, \eta$, and $\phi$ meson productions; (ii) direct $\gamma$-hadron correlations and single direct photon production; (iii) inclusive $J / \psi$ and $b \bar{b}$ pair productions; and (iv) azimuthal anisotropy in small collision systems.

\section{Light Flavor Production}

\section{$2.1 \pi^{0}$ and $\eta$ productions}

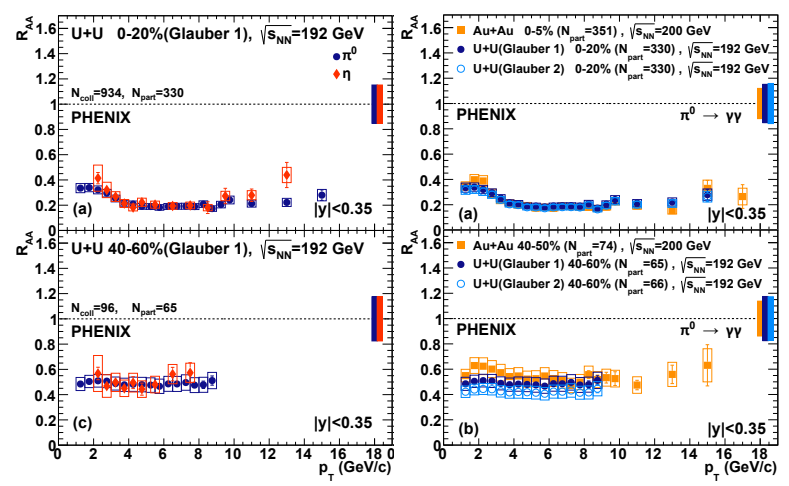

Figure 1. Nuclear modification factor $R_{A A}$ as a function of $p_{T}$ for $\pi^{0}$ and $\eta$ in $\mathrm{U}+\mathrm{U}$ collisions at $\sqrt{s_{N N}}=192 \mathrm{GeV}$.

Figure 1 shows the first measurements of the nuclear modification factor $R_{A A}$ for $\pi^{0}$ and $\eta$ mesons in $\mathrm{U}+\mathrm{U}$ collisions at $\sqrt{s_{N N}}=192 \mathrm{GeV}$ [1]. The $R_{A A}$ of $\pi^{0}$ and $\eta$ is measured to

\footnotetext{
*e-mail: todoroki@bnl.gov
} 
be consistent each other within uncertainties over the wide transverse momentum $\left(p_{T}\right)$ range, indicating the similar suppressions of $\pi^{0}$ and $\eta$ in QGP medium. A comparison of $\pi^{0} R_{A A}$ between $\mathrm{U}+\mathrm{U}$ and $\mathrm{Au}+\mathrm{Au}$ collisions made at similar numbers of participant nucleons shows the suppression depends only on the medium size regardless of collision systems.

\section{$2.2 \phi$ production}
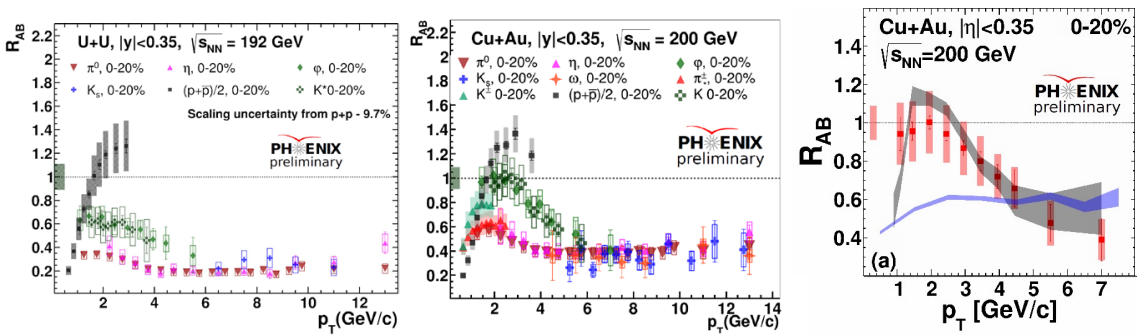

Figure 2. Nuclear modification factor $R_{A A}$ for $\phi$ meson in (left) $\mathrm{U}+\mathrm{U}$ collisions at $\sqrt{s_{N N}}=192 \mathrm{GeV}$ and (middle and right) $\mathrm{Cu}+\mathrm{Au}$ collisions at $\sqrt{s_{N N}}=200 \mathrm{GeV}$. The grey and purple bands in the right plot show AMPT with string melting and PYTHIA8 calculations, respectively.

Measurements of $R_{A A}$ are now extended to $\phi$ meson for $\mathrm{U}+\mathrm{U}$ and $\mathrm{Cu}+\mathrm{Au}$ collisions as shown in Fig. 2. In both collisions, $R_{A A}$ of various hadrons follows one universal curve at high- $p_{T}$ probably due to flavor-independent energy loss in the medium. In contrast, at intermediate $p_{T}$, those hadrons show a hierarchy of $R_{A A}(p \bar{p})>R_{A A}\left(\phi, K^{*}\right)>R_{A A}\left(\pi^{0}, \eta\right)$, which can be taken as an interplay of radial flow, strangeness enhancement, and quark coalescence effects. The importance of coalescence effect in $\phi$ meson production is supported by the better description of $R_{A A}$ by AMPT with coalescence than PYTHIA without coalescence.
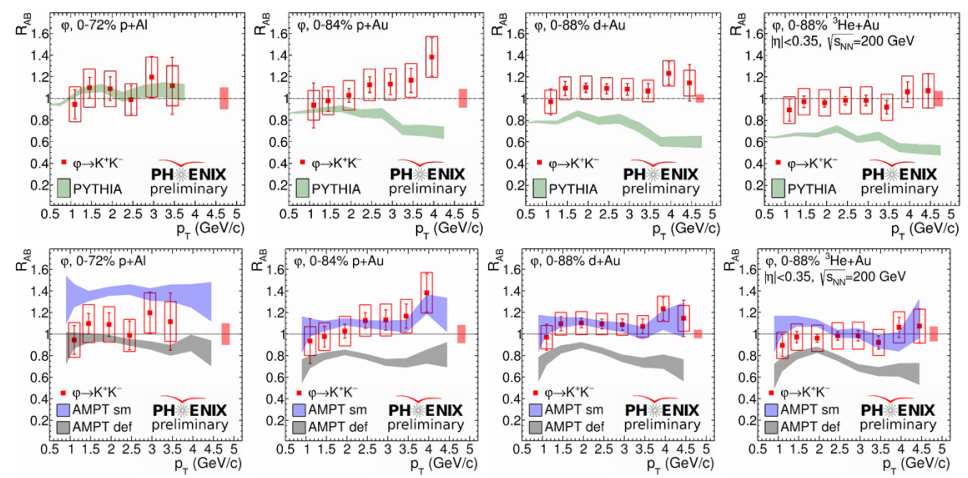

Figure 3. Nuclear modification factor $R_{A B}$ for $\phi$ meson in $p+\mathrm{Al}, \quad p+\mathrm{Au}$, $d+\mathrm{Au}$, and ${ }^{3} \mathrm{He}+\mathrm{Au}$ collisions compared with (top) PYTHIA and (bottom) AMPT calculations.

The $\phi$ meson production in small collision systems is investigated by measuring $R_{A B}$ as presented in Fig. 3. From $p+\mathrm{Au}$ to ${ }^{3} \mathrm{He}+\mathrm{Au}$ collisions, $R_{A B}$ is well described by AMPT with coalescence. While, in $p+\mathrm{Al}$ collisions, PYTHIA and AMPT without coalescence effects better describe the data. This implies that the QGP medium does not form in $p+\mathrm{Al}$ collisions. 

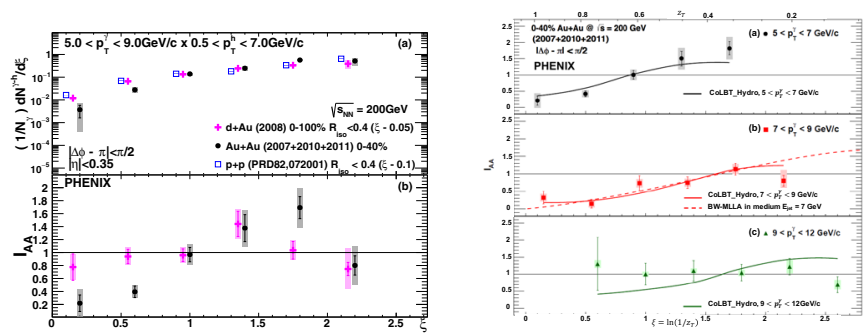

Figure 4. (Left-Top) Correlated yields and (Left-Bottom and Right) their ratios to the $p+p$ baseline $I_{A A}$ as a function of $\xi=\ln \left(p^{\gamma} / p^{h \pm}\right)$ in $p+p$, $d+\mathrm{Au}$, and $\mathrm{Au}+\mathrm{Au}$ collisions at $\sqrt{s_{N N}}=200 \mathrm{GeV}$.

\section{Hard Probes}

\section{1 $\gamma$-hadron correlations}

In-medium parton energy-loss mechanism is studied via direct $\gamma$-hadron correlations in $\mathrm{Au}+\mathrm{Au}$ collisions at $\sqrt{S_{N N}}=200 \mathrm{GeV}$. Figure 4 shows the ratio of correlated yields $I_{A A}$ between $\mathrm{Au}(d)+\mathrm{Au}$ and $p+p$ collisions. In $d+\mathrm{Au}$ collisions, $I_{A A}$ shows no modification from the $p+p$ baseline. However, in Au+Au collisions, $I_{A A}$ shows a suppression at low $\xi=\ln \left(p^{\gamma} / p^{h \pm}\right)$ (high $p_{T}$ ) and an enhancement at high $\xi\left(\right.$ low- $p_{T}$ ), which can be understood as parton energy loss at high $p_{T}$ and its redistribution at low $p_{T}$. This observation can be quantitatively reproduced by the CoLBT_Hydro model calculation [3].

\subsection{Direct photon production}
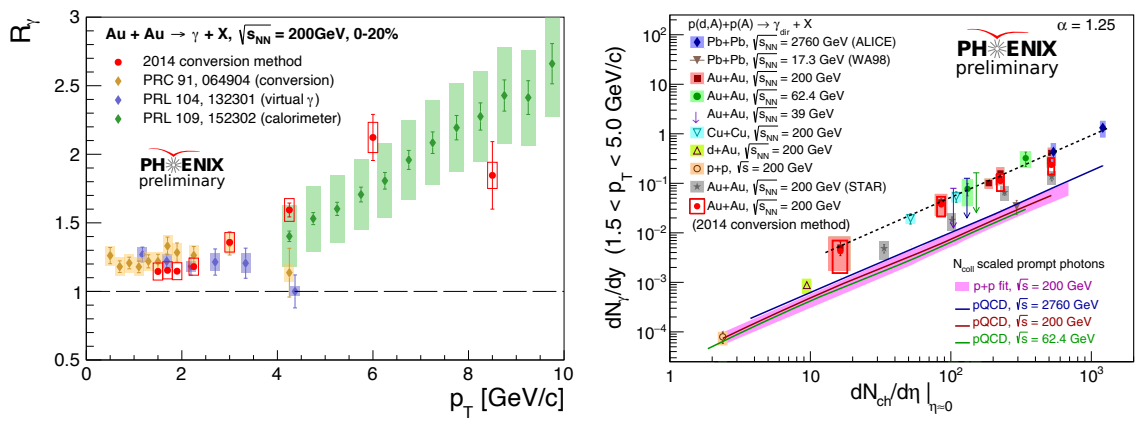

Figure 5. (Left) The direct photon fraction $R_{\gamma}$ as a function of $p_{T}$ and (Right) the integrated direct photon yield as a function of charged particle multiplicity in $\mathrm{Au}+\mathrm{Au}$ collisions at $\sqrt{s_{N N}}=200 \mathrm{GeV}$.

Single direct photon measurement has been updated using high luminosity Au+Au collision data set from the 2014 data taking period as shown in Fig. 5. The new measurement of direct photon fraction $R_{\gamma}$ with the external conversion method reconfirms the previous measurements with different methods over the wide $p_{T}$ range, significantly reducing the systematic uncertainties at high $p_{T}$. The integrated direct photon yield from this new measurement is also in excellent agreement with previous results.

\subsection{Heavy quark production}

Figure 6 shows the cross sections of inclusive $J / \psi$ as a function of $p_{T}$ in $p+p$ collisions at $\sqrt{s}=200$ and $510 \mathrm{GeV}$ in mid- and forward-rapidities. In $p_{T}>2 \mathrm{GeV} / c$, the $J / \psi$ production can be successfully described by the NLO+NRQCD calculations $[6,7]$ in both rapidi- 

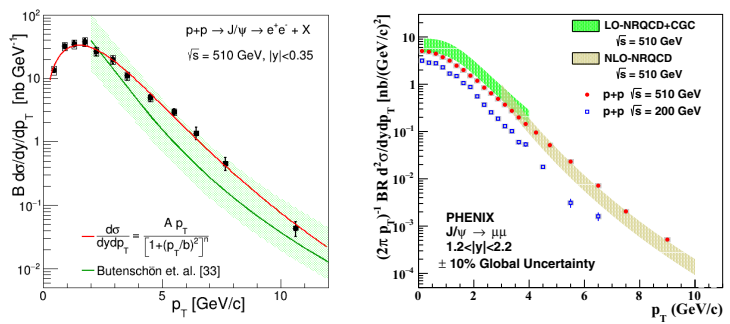

Figure 6. Cross section of $J / \psi$ as a function of $p_{T}$ and rapidity in $p+p$ collisions at $\sqrt{s}=200$ and $510 \mathrm{GeV}$ in (left) mid- and (right) forward-rapidities.

ties. Below $p_{T}=2 \mathrm{GeV} / c$, the $J / \psi$ production in forward-rapidity is overestimated by the $\mathrm{LO}+\mathrm{NRQCD}+\mathrm{CGC}$ calculations $[7,8]$.
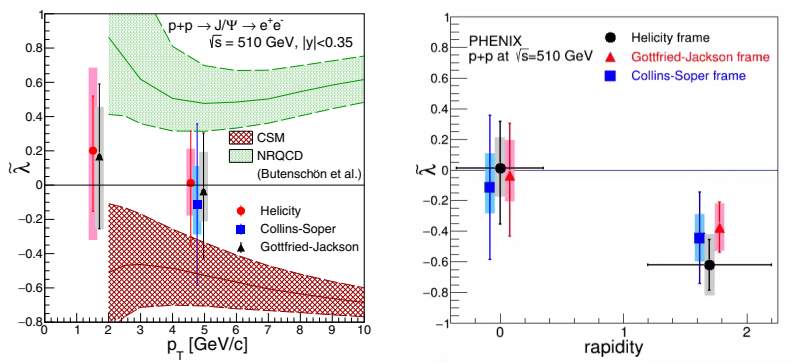

Figure 7. The frame invariant polarization $\tilde{\lambda}$ of $J / \psi$ in the Helicity, Collins-Soper, and Gottfried-Jackson in $p+p$ collisions at $\sqrt{s}=510 \mathrm{GeV}$ as a function of (left) $p_{T}$ and (right) rapidity.

The $J / \psi$ production mechanism has also been studied via measurements of the frame invariant polarization $\tilde{\lambda}$ in $p+p$ collisions at $\sqrt{s}=510 \mathrm{GeV}$ as shown in Fig 7. At midrapidity, $\tilde{\lambda}$ is consistent with zero within large uncertainties over the entire $p_{T}$ range. The calculations of NLO+NRQCD and color-singlet models are consistent with the data within large uncertainties. Unlike the mid-rapidity results, $\tilde{\lambda}$ shows a negative value in forwardrapidity, indicating longitudinal polarization.
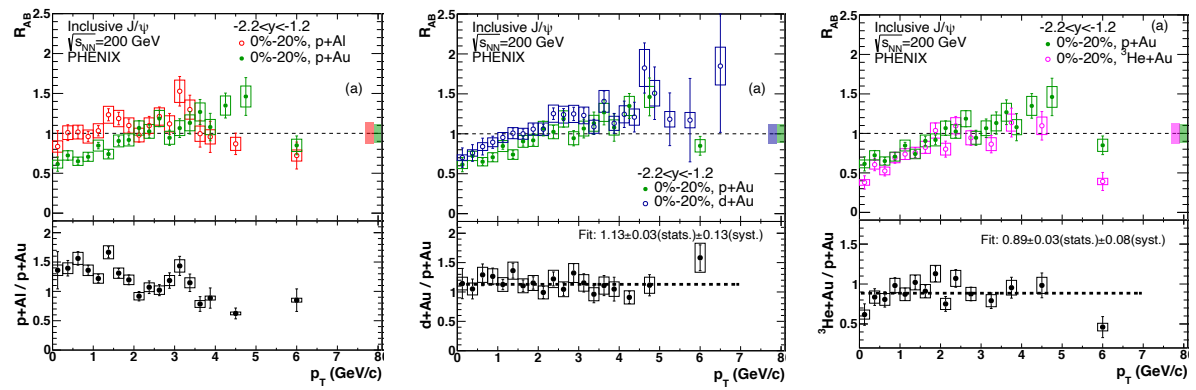

Figure 8. Nuclear modification factor $R_{A B}$ of inclusive $J / \psi$ in $p+\mathrm{Al}, p+\mathrm{Au}, d+\mathrm{Au}$, and ${ }^{3} \mathrm{He}+\mathrm{Au}$ collisions at $\sqrt{s_{N N}}=200 \mathrm{GeV}$.

Cold nuclear matter effects in the inclusive $J / \psi$ production are studied via the $R_{A B}$ measurements in $p+\mathrm{Al}, p+\mathrm{Au}, d+\mathrm{Au}$, and ${ }^{3} \mathrm{He}+\mathrm{Au}$ collisions at $\sqrt{s_{N N}}=200 \mathrm{GeV}$ [9] as shown in Fig. 8. In $p+\mathrm{Al}$ collisions, $J / \psi R_{A B}$ shows little suppression probably due to its small target size. From $p+\mathrm{Au}$ to ${ }^{3} \mathrm{He}+\mathrm{Au}$ collisions, $J / \psi R_{A B}$ is similar despite the increased energy density, indicating that cold-nuclear matter effects are dominant. 

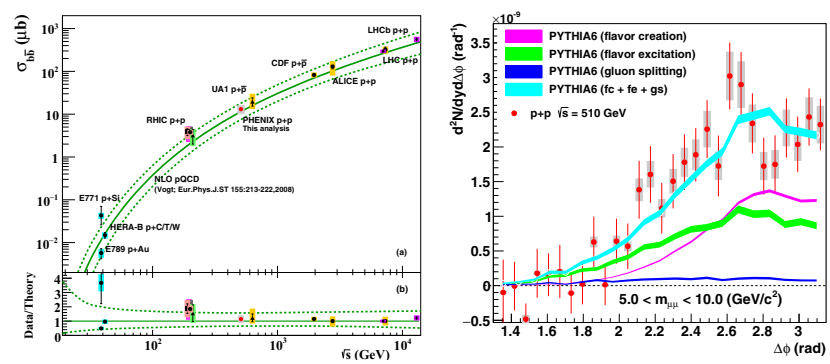

Figure 9. (Left) The production rate of $b \bar{b}$ pairs and (Right) relative angular distribution between $b \bar{b}$ pair quarks in $p+p$ collisions at $\sqrt{s}=510 \mathrm{GeV}$.

Shown in Fig. 9 is the production rate of $b \bar{b}$ pairs obtained via model dependent extrapolations from the yield of high-mass like-sign muons from neutral B meson oscillations in $p+p$ collisions at $\sqrt{s}=510 \mathrm{GeV}$ [10]. The production rate was estimated to be $\sigma_{b \bar{b}}=13.1 \pm 0.60$ (stat.) \pm 1.5 (sys.) \pm 2.7 (global). This new result follows the worldwide trend and is successfully described by pQCD calculations. A comparison of the relative angular distribution between $b \bar{b}$ pair quarks to PYTHIA calculations favors a dominant mixture of flavor creation and excitation over the gluon splitting.

\section{Flow in small systems}

As scientific due diligence, PHENIX revisited the measurements of second- and third-order azimuthal anisotropies $v_{2}$ and $v_{3}$ in high-multiplicity $p+\mathrm{Au}, d+\mathrm{Au}$, and ${ }^{3} \mathrm{He}+\mathrm{Au}$ collisions at $\sqrt{S_{N N}}=200 \mathrm{GeV}$ using the $3 \times 2 \mathrm{PC}$ method, taking the advantage of the event-mixing technique in canceling possible detector and beam optics effects [12]. In this $3 \times 2 \mathrm{PC}$ method, we measure two-particle correlations of three different sets of pairs. The $n$-th order oscillation $c_{n}$ extracted from those correlations is then combined to obtain single particle azimuthal anisotropy as $v_{n}^{A}=\sqrt{\frac{c_{n}^{A B} c_{n}^{A C}}{c_{n}^{B C}}}$, where $A, B$, and $C$ stand for sub-events used to construct those correlations. As was done in the PHENIX results published in Nature Physics using the EP method [11], we used BBCS(-3.9 $<\eta<-3.1)$, FVTXS $(-3<\eta<-1)$, and $\operatorname{CNT}(|\eta|<$ $0.35)$ detectors to measure mid-rapidity $v_{2}$ and $v_{3}$. Also as a systematic study, we employed FVTXS $(-3<\eta<-1), \operatorname{CNT}(|\eta|<0.35)$, and FVTXN $(1<\eta<3)$ detectors.
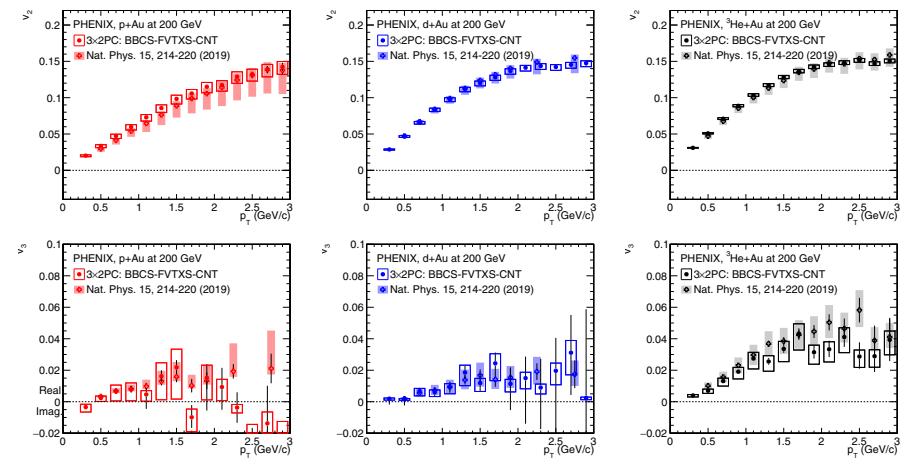

Figure 10. Measurements of (top panels) $v_{2}$ and (bottom panels) $v_{3}$ with the $3 \times 2 \mathrm{PC}$ method by the BBCS-FVTXS-CNT detector combination in highmultiplicity $p+\mathrm{Au}, d+\mathrm{Au}$, and ${ }^{3} \mathrm{He}+\mathrm{Au}$ collisions at $\sqrt{s_{N N}}=200 \mathrm{GeV}$.

Figure 10 shows measurements of $v_{2}$ and $v_{3}$ with the $3 \times 2 \mathrm{PC}$ method by the BBCSFVTXS-CNT detector combination. Both $v_{2}$ and $v_{3}$ are in excellent agreement with PHENIX results published in Nature Physics, which reconfirms the robustness of the previous measurements. 

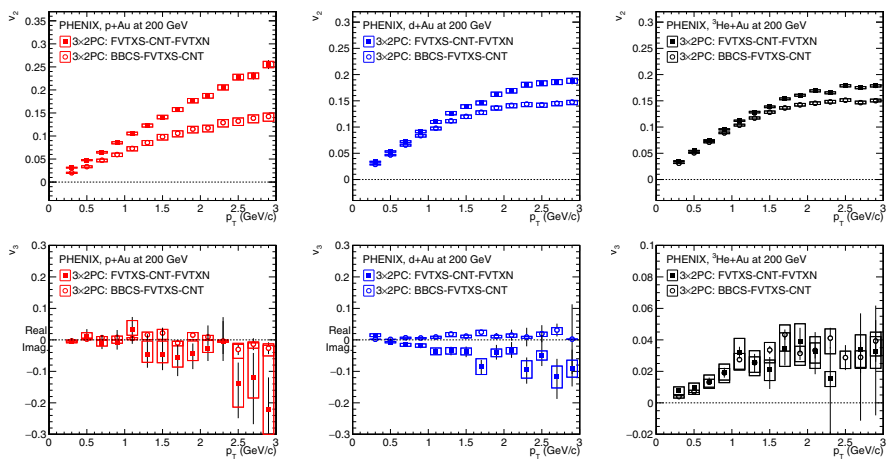

Figure 11. Measurements of (top panels) $v_{2}$ and (bottom panels) $v_{3}$ with the $3 \times 2 \mathrm{PC}$ method by the FVTXS-CNT-FVTXN detector combination in highmultiplicity $p+\mathrm{Au}, d+\mathrm{Au}$, and ${ }^{3} \mathrm{He}+\mathrm{Au}$ collisions at $\sqrt{s_{N N}}=200 \mathrm{GeV}$.

In contrast, measurements with the $3 \times 2 \mathrm{PC}$ method by the FVTXS-CNT-FVTXN detector combination show larger $v_{2}$ than that with the BBCS-FVTXS-CNT detector combination. This can be understood by stronger non-flow in the FVTN acceptance than BBCS acceptance due to smaller multiplicity in the FVTXN acceptance. Also, the wider rapidity gap between FVTXS and FVTXN than that between BBCS and FVTXS results in stronger event-plane decorrelations. Both of these changes by the kinematic selections push up $v_{2}$ values. This detector combination shows $v_{3}$ consistent with that with the BBCS-FVTXS-CNT detector combination in ${ }^{3} \mathrm{He}+\mathrm{Au}$ collisions, while it shows mostly imaginary $v_{3}$ in $p+\mathrm{Au}$ and $d+\mathrm{Au}$ collisions. This is probably because the stronger non-flow and the event-plane decorrelation effects in these systems overwhelm the small $v_{3}$ signals.

These systematic measurements warrant more experimental and theoretical studies to understand the non-flow and event-plane decorrelation effects in small collision systems.

\section{Summary}

In summary, we have presented highlight results from recent ample data sets for $p+p$, small, and large system collisions such as: (i) $\pi^{0}, \eta$, and $\phi$ meson productions; (ii) direct $\gamma$-hadron correlations and single direct photon productions; (iii) inclusive $J / \psi$ and $b \bar{b}$ pair productions; and (iv) azimuthal anisotropy in small collision systems. Many important analyses that use 36 billion events of $\mathrm{Au}+\mathrm{Au}$ collisions at $\sqrt{s_{N N}}=200 \mathrm{GeV}$ from 2014 and 2016 operations are underway and many exciting physics results are yet to come from the PHENIX Collaboration.

\section{References}

[1] U. Acharya et al. (PHENIX Collaboration), Phys. Rev. C 102, 064905 (2020)

[2] U. Acharya et al. (PHENIX Collaboration), Phys. Rev. C 102, 054910 (2020)

[3] W. Chen et al., Phys. Lett. B 777, 86-90 (2018)

[4] U. Acharya et al. (PHENIX Collaboration), Phys. Rev. D 101, 052006 (2020)

[5] U. Acharya et al. (PHENIX Collaboration), Phys. Rev. D 102, 072008 (2020)

[6] M. Butenschoen et al., Phys. Lev. Lett. 106, 022003 (2011)

[7] Y. Ma et al., Phys. Lev. Lett. 106, 042002 (2011)

[8] Y. Ma et al., Phys. Lev. Lett. 113, 192301 (2014)

[9] U. Acharya et al. (PHENIX Collaboration), Phys. Rev. C 102, 014902 (2020)

[10] U. Acharya et al. (PHENIX Collaboration), Phys. Rev. D 102, 092002 (2020)

[11] C. Aidala et al. (PHENIX Collaboration), Nature Physics 15, 214 - 220 (2019)

[12] U. Acharya et al. (PHENIX Collaboration), arXiv:2107.06634 [hep-ex] 